\title{
Cultura enfermera alrededor de las prácticas profesionales efectuadas por estudiantes de enfermería
}

\section{The culture of nursing professionals about practices conducted bynursing students}

\section{A cultura dos profissionais de enfermagem sobre as práticas realizadas por estudantes de enfermagem}

${ }^{1}$ Mitzi Letelier Valdivia; ${ }^{2}$ José Siles González; ${ }^{3}$ Ana Luisa Velandia Mora.

\author{
${ }^{1}$ Licenciada en Enfermería y Magister en Gerontología Social. \\ Profesor Investigador U. de los Andes, Santiago de Chile. Doctoranda U. de Alicante. \\ ${ }^{2}$ Doctor en Historia, Licenciado en Pedagogía y Diplomado en Enfermería.
}

Catedrático de la E.U. de Enfermería. U. de Alicante.

${ }^{3}$ Doctora en Ciencias Médicas, con mención en Salud Pública. Instituto de Medicina Sanitaria de San Petersburgo, Rusia. Profesora Emérita, Universidad Nacional de Colombia.

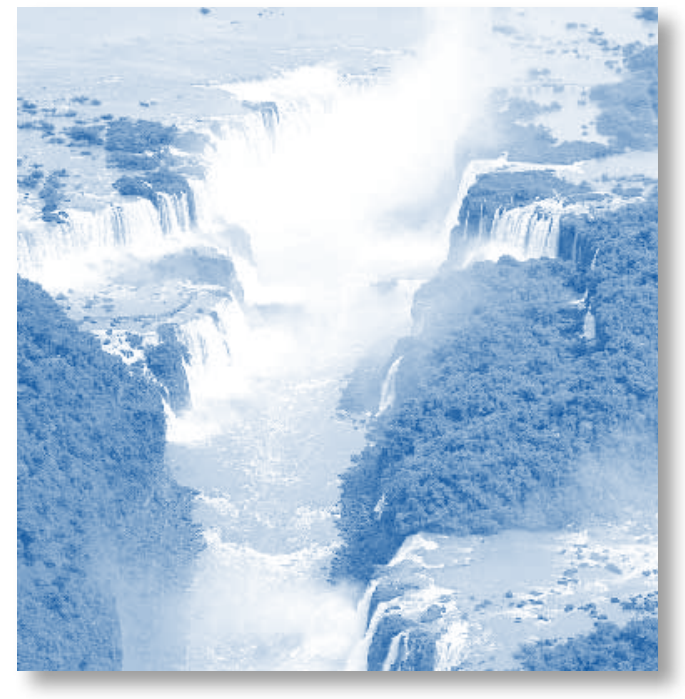

\section{ABSTRACT}

Course 2010/2011 ten students of nursing at the University of Alicante were sent to nursing EU countries to conduct their professional practices. This raises the concern to understand the nurse culture in the context of the European Union from the experience of nursing students Erasmus. Material and Methods. Ethnography. Strategy of data collection: observation. Role of non-participant observer researcher. Informant: Erasmus student nurs- es 2010/2011 academic year. Sources: Written (field notes). Ethical issues. Consent is sought for teachers and students to attend coordination meeting and informative. Descriptive analysis by Boyle (1994). Results: The data reported characteristics of the context of learning, esenario practices, practical experience of students and caregivers practices in the various scenarios. Subcategories are identified as a mentor, teaching methods, range, type of practical work, learning, practice carers. Conclusion: The professional culture in the context of the Union remains diverse in terms of the level of training and practice carers. As components of culture emerge the importance of mentor figure for its proximity to the student and identifies contextual features that promote student learning.

Keywords: Cultural Characteristics, Education Nursing, Faculty Practice Nursing, Practical Nursing, Professional Training.

\section{RESUMO}

No período letivo 2010 / $2011 \mathrm{dez}$ alunos de enfermagem foram enviados levanta da $U$. 
da Universidade de Alicante para países da União Europeia para realizar suas práticas profissionais. Isto levanta a preocupação de entender a cultura enfermeira no contexto da União Europeia a partir da experiência de enfermagem estudantes Erasmus. Material e Métodos. Etnografia. Estratégia de coleta de dados: observação. Papel das organizações não-participante pesquisador observador. Informante: estudante Erasmus enfermeiras ano lectivo 2010/2011. Fontes: Escrito (notas de campo). Questões éticas. Consentimento é procurado por professores e alunos para assistir à reunião de coordenação e informativo. Análise descritiva por Boyle (1994). Resultados: Os dados reportados características do contexto de aprendizagem e ambiente profissional, onde eles fazem a prática clínica. Subcategorias são identificados como um mentor, métodos de ensino, série, tipo de trabalho prático, de aprendizagem, os prestadores de cuidados prática. Conclusão: A cultura profissional no contexto da União continua diversificada em termos do nível de encarregados de educação treinamento e prática. Como componentes da cultura emerge a importância da figura do mentor de sua proximidade com o aluno e identifica os recursos contextuais que promovem o aprendizado do aluno.

Palavras-chave: Características Culturais, Educação em Enfermagem, Prática do Docente de Enfermagem, Enfermagem Prática, Capacitação Profissional.

\section{RESUMEN}

Del curso 2010/ 2011 diez estudiantes de enfermería de la Universidad de Alicante, fueron enviados a distintos países de la UE para realizar sus prácticas profesionales. Surge así la inquietud de comprender la cultura enfermera en el contexto de la Unión Europea desde la experiencia de los estudiantes de enfermería Erasmus. Material y Método. Etnografía. Estrategia recolección de datos: observación. Rol del investigador: observador no participante. Informantes: estudiantes de enfermería Erasmus del curso 2010/2011. Fuentes: Escrita (notas de campo). Aspectos éticos. Se solicita consentimiento a profesores y alumnos para asistir a reunión de coordinación e informativa. Análisis del tipo descriptivo según Boyle, (1994). Resultados: los datos informan características del contexto del aprendizaje, del escenario de las prácticas, la experiencia práctica de los alumnos y de las prácticas cuidadoras en los diversos escenarios. Se identifican sub categorías como mentor; métodos de enseñanza, autonomía; tipo de trabajo práctico, aprendizajes; practicas cuidadoras. Conclusión: La cultura profesional en el contexto de la Unión sigue siendo diversa en cuanto al nivel de formación y prácticas cuidadoras. Como componentes de la cultura emerge la importancia de la figura del mentor por su cercanía con el estudiante y se identifican características del contexto que favorecen el aprendizaje del estudiante.

Palabras Clave: Características Culturales, Educación en Enfermería, Práctica del Docente de Enfermería, Enfermería Práctica, Capacitación Profesional.

\section{INTRODUCCIÓN}

La experiencia de movilidad geográfica e intercambio como parte de los programas de Enseñanza Superior en Enfermería no es algo nuevo, tal como lo pone en evidencia el documento no 199 de la OMS publicado en 1960.

La Enseñanza Superior de Enfermería en España a partir de la integración de los estudios en la Universidad (1977) supuso un paso adelante en su proceso de profesionalización; 
es decir, en la transición de la disciplina enfermera desde una etapa técnica a una profesional (Martínez, 2007). Más tarde, en la década de los 90, el proceso de cambio que sufre la Universidad (Real Decreto 1231/2001) permite a los estudiantes alcanzar un mayor protagonismo en el proceso de enseñanza-aprendizaje. Así, los estudios de la diplomatura en enfermería empiezan a estar en consonancia con la normativa europea que propugna la libre circulación y el intercambio de profesores y estudiantes en el marco comunitario.

Esta nueva oportunidad en el desarrollo de la profesión y de los futuros profesionales se cristaliza en 1998 a través de lo que se ha denominado Programa Erasmus. El "Programa Erasmus" es el programa más destacado e importante por su éxito y sus repercusiones, siendo el embrión y origen del actual proceso de integración del Espacio Europeo de Educación Superior [EEES] y la Convergencia de las universidades de la Unión Europea [UE]. Y esta convergencia ha dado paso al nuevo Programa de Aprendizaje Permanente (Lifelong Learning Programme), que sigue integrando la acción Erasmus. Entonces, la creación del Espacio Europeo de Educación Superior (Ministerio de Educación, Cultura y Deporte, 2003) en este nuevo milenio, supone una oportunidad única para la mejora de la calidad de la enseñanza superior en toda Europa, abriendo también una nueva etapa para la formación en Enfermería con el establecimiento desde el 2003 del Sistema Europeo de Créditos [European Credits Transfer System -ECTS-] (Real Decreto 1125/2003), el Suplemento Europeo al Título (Real Decreto 1044/2003) y la nueva estructura de los estudios de Grado (Real Decreto 55/2005) y Posgrado (Real Decreto 56/2005) en el 2005. Hechos todos que han contribuido a modificar el escenario de la

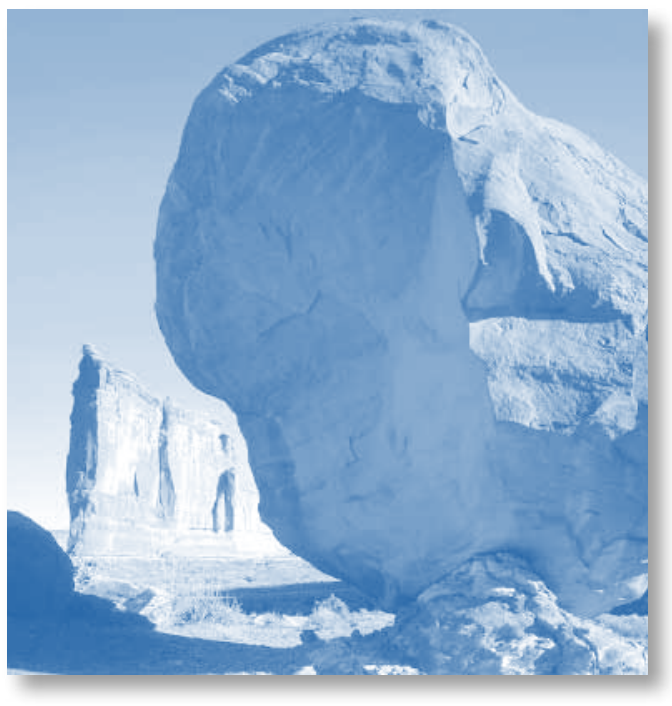

educación en enfermería a partir de la reformulación de planes de estudios y metodologías sobre la base de un aprendizaje orientado desde los nuevos paradigmas educativos de la sociedad del conocimiento y de la información: innovación docente y aprendizaje a lo largo de la vida (Libro Blanco, 2005).

\section{Contexto Prácticas Clínicas de Enfermería en la U. de Alicante.}

El período ordinario de realización de prácticas clínicas corresponde a la matrícula de un curso completo y consta de 4 módulos clínicos de 4 semanas de duración cada uno de ellos, a realizar en los meses de enero a mayo, en turnos rotativos semanalmente (turno en horario de mañana, de 8 a 15 horas y en horario de tarde, de 15 a 22 horas). En el período extraordinario de ejecución de prácticas clínicas, realizado de modo excepcional y por causas justificadas, el estudiante puede realizar como máximo 2 módulos más por curso académico (de acuerdo con su matrícula), a cumplir en el periodo extraordinario de verano (junio - julio). La Comisión de Prácticum del Centro, al finalizar el primer cuatrimestre, en función de la solicitud del estudiante, de los re- 
cursos sanitarios disponibles y de los objetivos docentes, establecerá la asignación a los estudiantes. Esta planificación es publicada por la Escuela, presentada a los centros asistenciales, antes del inicio del período clínico, en virtud del convenio de colaboración vigente. Es decir, los estudiantes deben realizar 12 módulos clínicos para toda la titulación.

\section{Contexto del estudiante del Programa Eras- mus de Enfermería.}

La Universidad de Alicante dentro del Programa de Aprendizaje Permanente (Lifelong Learning Programme) mantiene "acuerdos Erasmus" con 383 instituciones de Educación Superior, entre las que continúan destacando las de Francia, Alemania, Italia y Reino Unido. Y le siguen en orden de importancia Portugal, Bélgica y Finlandia. Además, como reconocimiento a la tarea realizada por la universidad en el fomento y difusión de las actividades que apoya el Programa Erasmus, la Comisión Europea le ha concedido la Carta Universitaria Ampliada, que establece el marco general para las actividades de cooperación a nivel europeo que un centro de enseñanza superior puede realizar en el marco del programa Erasmus como parte del Programa de Aprendizaje Permanente (PAP). La Carta Universitaria Erasmus debe concederse como requisito previo para que el centro de enseñanza superior organice la movilidad de los estudiantes y el personal docente y de otro tipo, imparta los cursos intensivos de idiomas y los programas intensivos de Erasmus, y presente su candidatura para proyectos multilaterales, redes y medidas de acompañamiento y organice visitas preparatorias. La Carta Universitaria Erasmus se basa en la Decisión relativa al PAP (Decisión $\left.\mathrm{n}^{\circ} 1720 / 2006 / \mathrm{CE}\right)$, que abarca el período de 2007 a 2013. Los objetivos específicos del PAP figuran en el artículo 1, apartado 3, de dicha Decisión (Diario oficial UE del 31.03.2011)

En la Diplomatura en Enfermería la experiencia de intercambio a través del Programa Erasmus tiene diez años de trayectoria, y a través de él la/os estudiantes de tercer nivel se trasladan a distintos países de la Unión para realizar su programa de prácticas clínicas. Dichas experiencias están integradas en las asignaturas del Plan de Estudios y se realizan durante el segundo cuatrimestre en centros clínicos asistenciales.

En el caso de los estudiantes Erasmus, tras cumplir una serie de requisitos de excelencia académica y dominio del inglés [B1], acceden a cursar su practicas clínicas en instituciones de salud de otro país de la Unión Europea suscrito a convenio con el Departamento de Enfermería de la Universidad de Alicante. Además, una vez seleccionados, deben completar una serie de otros requisitos que varían de acuerdo al país y la institución de destino. Así por ejemplo, todos deben preparar y entregar los denominados "papeles de movilidad" que conforman el contrato de subvención y compromiso del alumno. También, deben rellenar el "formulario de solicitud" y el "acuerdo de aprendizaje" los cuales deben ser completados en la institución que los acoge para permitir el reconocimiento de los estudios cursados a su regreso. A partir de aquí hay una serie de variaciones según el país de destino.

Para el Reino Unido el estudiante debe adjuntar un certificado de antecedentes y rendir un test psicológico, esto como requisito contemplado en las normativas que regulan el trabajo con personas. En otro orden de cosas se les solicitan una serie de exámenes clínicos relacionados con la certificación de los niveles de inmunidad para el bacilo de Koch, para el virus de Hepatitis B y otras enfermedades como 
la difteria, tétanos, meningitis entre otras; así como la prueba de SAM para determinar si son portadores del staphylococus aureus. Todas estas disposiciones se deben interpretar como parte de la existencia de una cultura de cuidados centrada en la protección al paciente. Además y siempre dentro del Reino Unido, se les suelen solicitar cartas de recomendación, medida que responde a las mismas políticas de seguridad de los cuidados. Por último, otros países como Alemania y Finlandia les suelen solicitar además un currículum vitae que considere también sus intereses personales, inclusive este último solicita una carta de motivaciones.

Lo anterior se complementa con una serie de formularios relativos a las ayudas económicas a las que pueden acceder los estudiantes de intercambio procedentes tanto de la Universidad como de sus respectivos Gobiernos Provinciales y Ayuntamientos. Estas ayudas contribuyen a costear los gastos de alojamiento y traslado en los cuales deben incurrir los estudiantes, por cuanto vivir la experiencia Erasmus, también supone un esfuerzo económico personal a los estudiantes y sus familias.

\section{Marco Teórico.}

Toda investigación debe estar enfocada dentro de un marco teórico. Su utilidad recae en aportar una lógica que ordena y enmarca desde un enfoque conceptual y teórico el fenómeno de estudio. Esto porque "ningún fenómeno es un hecho dispuesto para ser recogido. Se precisa pues elegir entre los fenómenos, entre los diferentes aspectos de un mismo fenómeno, y estas elecciones sólo pueden hacerse en función de un marco teórico" (Cresswell y Godelier, 1981:21)

En la actualidad, en el mundo científico impera la tendencia caracterizada por la op- cionalidad de paradigmas, estrategias de investigación y métodos de análisis, también por el descubrimiento y re-descubrimiento de modos de investigación cualitativa junto al reconocimiento de la actividad investigativa como un proceso multicultural. Entonces, estos hechos reflejan que la necesaria coexistencia de los distintos tipos de paradigmas es un signo de la presencia de las distintas tradiciones científicas que co - existen hoy.

El paradigma es concebido como el sistema de creencias básicas, principios o supuestos de carácter ontológico [naturaleza de la realidad estudiada], epistemológico [modelo de relaciones entre el investigador y lo investigado] y metodológico [modo de obtener conocimiento de la realidad]. Y todos ellos están relacionados entre sí, de modo que la creencia básica asumida por el investigador en lo ontológico, le lleva a adoptar posturas consonantes con cada uno de los elementos descritos; es decir, una posición onto-epistemológica (Guba y Lincoln, 1985, 90,94:113-145). Y desde la necesaria producción de conocimiento también desde la disciplina de enfermería, investigar exige determinar el paradigma con que se mirará esa realidad, tener presente la situación y el tema de estudio (Cabrero J y Richart M, 1996).

En esta monografía el fenómeno al cual se intenta proporcionar sentido y legitimación es a la experiencia cultural según la cual un grupo de alumnos de enfermería se aproximan a la cultura enfermera en un contexto y escenario de prácticas fuera de su país, pero dentro del marco del Espacio Europeo de Educación Superior. Este hecho es importante de investigar porque las ciencias humanas o sociales buscan comprender el significado, los valores y la intencionalidad del ser humano autor de la acción. Para ello no olvida las diferencias 


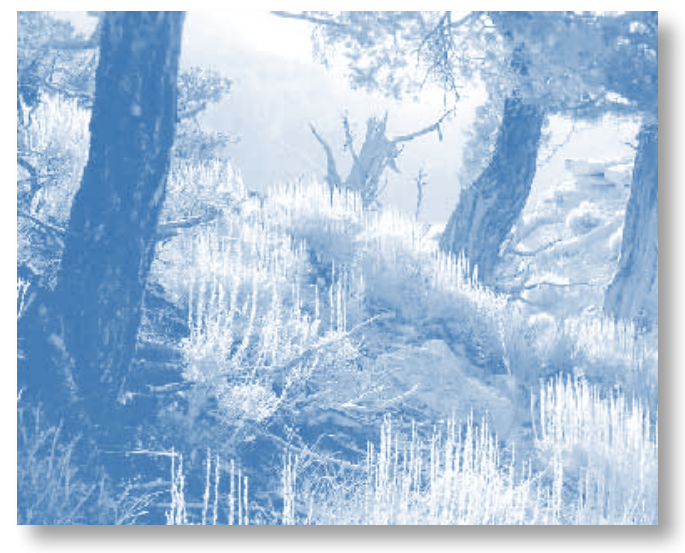

existentes entre los sujetos a la luz de sus respectivos contextos históricos sociales. (Arnal, del Rincón, y Latorre, 1992: 12), (Hesse, 1991:105).

Esta situación lleva a los investigadores a plantearse la aplicación de la perspectiva hermenéutica entendida esta desde su origen lingüístico [hermeneutiké téchne, ars interpretationis] como el arte de la interpretación que debe su cualificación tradicional a la dimensión práctica del término -Hermes- [el mensajero de los dioses] y como tal, alude a transformación. Sin embargo, esta palabra alcanza una plena comprensión cuando se reemplaza la anterior expresión griega por hermenéia, en palabras de (Kerényi, K., 1963:133-4) “[...] alfa y omega de la hermenéutica" (Ferraris, Maurizio, 1988:9). Desde la hermenéutica en la actualidad, se plantea que no es posible inteligir -algo- a no ser a través de símbolos y textos donde éstos últimos, siempre permiten nuevas lecturas. Por tanto, la interpretación no acaba en el texto, sino que se abre a la configuración de mundos más humanos. Este tipo de conocimiento se fundamenta en una concepción de hombre espiritual e histórico y toma como modelo de partida a la estética porque lo que está en juego es el obrar humano y la felicidad del hombre, posición asumida en esta investigación.
Esto viene a significar que las ciencias sociales pueden investigar experiencias vividas [concepto de Erlebnis] por las personas, relacionándolas con su contexto socio-histórico y cultural (1980:82 leído en Valles, 2007: 24) y donde el acto de conocer no es distinto del objeto conocido, (Ferraris, M.,1988,2000:153). $\mathrm{Al}$ respecto se plantea que la -vida misma- se autointerpreta, tiene estructura hermenéutica (Gadamer, 1960), por tanto, es la base de las ciencias del espíritu (Ferraris, M.,1988,2000:150).

\section{Marco Metodológico.}

Con este marco, se plantea como objetivo para este estudio: Describir las características de la cultura enfermera en torno a las prácticas profesionales de los estudiantes Erasmus de la Universidad de Alicante, en el escenario Europeo de Educación Superior, en Enfermería.

Para ello y desde la perspectiva de la realidad que abarca el fenómeno de estudio se plantea un diseño cualitativo con enfoque etnográfico. En primer lugar la etnografía se basa en el trabajo de campo, rúbrica de la antropología cultural, ciencia que estudia los grupos humanos a través de su cultura, "es un campo sistemático de estudio o campo de conocimiento que intenta, a través de la experimentación, la observación y la deducción, dar explicaciones fiables de un fenómeno, con referencia al mundo material y físico" (Prentice - Hall,inc, 1993:937). Así, el trabajo etnográfico se caracteriza por su talante holístico, y se ocupa de estudiar las formas en que la cultura afecta a la experiencia individual, o en ofrecer una visión global del conocimiento, de las costumbres y de las instituciones de un pueblo (Barfield, 1997, 2000:43).

Entonces, a diferencia de otras ciencias sociales, la antropología ofrece una perspec- 
tiva transcultural a través de la comparación constante de costumbres de una sociedad con otra (Kottak, 2006:4) Es decir, cultura entendida como el conjunto de posibilidades de información de una sociedad (García Marco, 1995:113). A su vez, las sociedades humanas, informadas conforme a los parámetros culturales, cada vez dependen más de la cultura para su reproducción y su adaptación al entorno, al ser este entorno cada vez más, producto de la acción del hombre (García Marco, 1995:113). Entonces, el conocimiento del fenómeno de estudio requiere de una base biológica (ser humano) y cultural (contexto), sin la cual no puede transmitirse ni utilizarse la información. Por ello, en este estudio se considera tanto a los sujetos (alumnos Erasmus) como al escenario (Unión Europea) donde ocurre la transmisión de información, es decir, Instituciones de Salud vinculadas a los Departamentos de Enfermería en los países de Portugal, Reino Unido y Finlandia.

En tanto la cultura enfermera "es el todo complejo estructurado de elementos [objetos materiales, actos corporales, ideas, valores, sentimientos] y hechos culturales, que abarcan extensas áreas de la vida del ser humano, los cuales son aprendidos o pueden aprenderse y por tanto se transmiten social y mentalmente más que biológicamente entre generaciones que comparten una cultura (...)" (Letelier, 2011).

\section{MATERIAL Y MÉTODO}

\section{Trabajo de campo.}

La Estrategia de recolección datos considera como técnica la observación, herramienta etnográfica por excelencia. El rol del investigador es el de observador no participante. Los informantes son diez estudiantes enviados durante el curso 2010/2011 a Lisboa, Aberdeen, Newcastle, Glasgow, Hyvinkaa a través del
Programa Erasmus. Las Fuentes de donde se extraen los datos son escritas (notas de campo) y documentales (memoria de los estudiantes). La validez interna del estudio, está sujeta a la pertinencia teórica y práctica dada por la actualidad el tema y por la accesibilidad al campo e informantes. La necesidad de negociar un marco de interacción con los actores se cautela a través de las consideraciones éticas, según las cuales se establece contacto con los estudiantes una vez gestionadas la autorización a nivel institucional y, en virtud de la regulación de confidencialidad de los datos personales de los estudiantes, se recurre a la figura del portero quien, antes de que el investigador entre en contacto con los estudiantes, solicita su consentimiento a los alumnos. De esta manera la investigadora puede asistir como observador no participante a una reunión de coordinación e informativa en la cual además acontecería la situación de intercambio de experiencias entre los estudiantes enviados el curso anterior 2009/2010 y los del curso actual 2010/2011, hecho de interés para este estudio. El control de la subjetividad se logra a través de la estrategia de comparar los datos obtenidos durante la observación con los datos registrados por los alumnos en las memorias que deben entregar como parte del compromiso que asumen con la organización del Programa Erasmus, el cual contempla una autorización previa para ser empleadas como material de consulta de profesores y alumnos de la universidad.

El análisis realizado es del tipo descriptivo (Boyle, 1994), porque los datos analizados son parte de una investigación más amplia aún en curso.

\section{RESULTADOS}

El discurso de los alumnos de enfermería Erasmus desvela las características del con- 
texto de aprendizaje que encontraron los estudiantes durante su experiencia de intercambio en distintos países y ciudades de la Unión Europea, durante el curso 2010/2011 y dan a conocer rasgos de las practicas cuidadoras en dichos entornos.

\section{Contexto del aprendizaje.}

Práctica docente: De los datos emerge la figura del mentor (Reino Unido) o tutor de prácticas: “(...) cada estudiante va con una mentor, vas con una enfermera y haces su turno". Esta persona es un profesional a juicio de los estudiantes con buena base teórica (Portugal): "Llegan hasta el fondo, mínimo detalle para que llegues al fondo de por qué haces las cosas" Para cumplir esta función se les libera de carga asistencial: "Tienen menos carga pues para que puedan enseñarnos”. Además, en opinión de los estudiantes su desempeño guarda relación con el hecho que son evaluados por sus tutelados: "Y se esfuerzan porque luego tú los evalúas".

Métodos de enseñanza: se desprenden de los métodos empleados por el mentor y que los estudiantes valoran positivamente porque se basan en la relación personal entre ambos: "están contigo"; "te acompañan", "se esfuerzan por que tú aprendas", "hacen hincapié en cosas que se nos escapaban". Además está el fomento de la autonomía: “(...) te dejan hacer todo lo que quieras hacer si te ven motivada, te ayudan en todo". Sin embargo esta realidad no es la misma en todos los escenarios: "un mes ayudando al ordenador (...) no me enseñaron nada, (...) primera visita, te pone con auxiliar".

Experiencia práctica de los estudiantes: sobre el tipo de trabajo práctico que realizan aquí las opiniones varían entre los estudiantes; "haces trabajo de auxiliar", "no puedes hacer nada, haces cursos y te dejan hacer cosas, ni

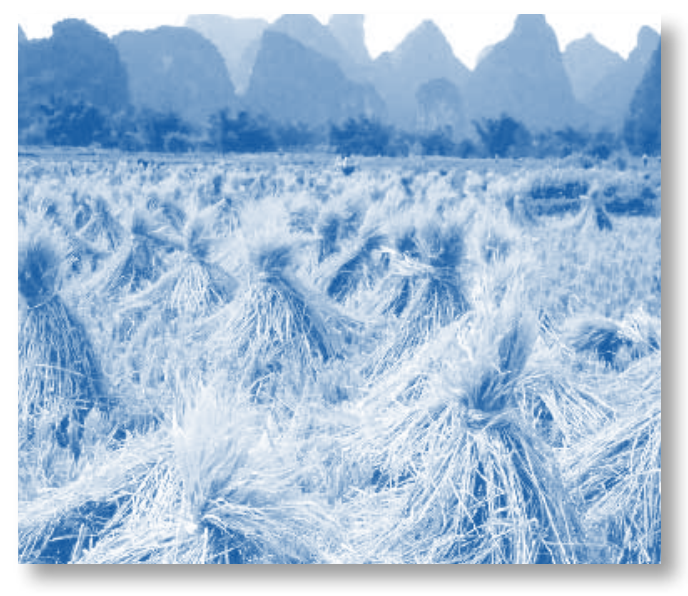

cambiar sueros" (RU) o "trabajo es el mismo" (Portugal); situación que revela las diferencias imperantes entre los países de la unión. En tanto la experiencia vivida aporta a los estudiantes algunos de los siguientes aprendizajes: "hay que ser súper puntual, yo decía 7.30 voy bien, pero tu llegabas 7.30 y estaban todas sentadas y luego me sentía súper mal que la gente llega 1 hora antes y si llegas tarde no se dice nada pero se quedan a recuperar", "cosas positivas que aprendes, cosas negativas que no hay que aprender". Sobre los lugares de las prácticas la opiniones también difieren: "yo súper contenta, (...) yo en la vida me he levantado 6 am para ir a trabajar e íbamos con una felicidad, yo me levantaba feliz", "todo es tan distinto que te cuesta acostumbrarte", "ves la mala pata que donde vayas a caer, la persona con que vayas a caer".

\section{Rasgos de las prácticas cuidadoras.}

Nivel de enfermería: los datos dejan traslucir diferencias en la formación del nivel técnico de enfermería que aún existe en el contexto de la unión. "No necesitan titulación y están allí porque les gusta” (Reino Unido), “(...) nos explicaron que como auxiliar llega sin preparación, formación todo lo hace la enfermera, nota que el cuidado integral se los das, se lo haces todo y no se te pasan las cosas" (Portugal). 
Distribución del trabajo: el equipo de enfermería cumple funciones y realiza actividades que también varían de un lugar a otro: "Personas de la limpieza que pulen el suelo pasan a hacer las camas, y con desinfectante limpian todas las mesas, las baranditas, los hierros de las camas, pastillas de desinfectante y lo haces tú para desinfectar todo", "allí todo el mundo hace de todo", "hay auxiliares que ponen vía (...)" (Reino Unido); “(...) todo lo hace la enfermera, nota que el cuidado integral se los das, se lo haces todo y no se te pasan las cosas" (Portugal); "enfermeras que se ocupan de la administración”, “(...) ponen rulos, preparan desayunos, la siesta una pasada” (Finlandia).

Practicas cuidadoras: los estudiantes identifican un tipo de cuidado más personalizado y centrado en la seguridad: " 15 pacientes por equipo, 3 enfermeras y 2 auxiliares" (Reino Unido); "se vuelcan sobre el paciente (...) que esté cómodo, todo lo que necesite, la almohada, oye no hay úlceras allí"; "no verás ni un abuelito que se levante y no salga una persona joven a ayudarlo" (Reino Unido) y centrado en la seguridad y bienestar del paciente: "hay tantas pastillas, firma tú, firma tú” (Reino Unido), “(...) en todas las habitaciones había lavado de manos y en la salida de cada habitación, para eliminar corto punzante. En Escocia no hay ni medio paciente que entre sin lavarse las manos, está lleno de carteles en todas partes "ahora tiene que lavarse las manos"; "yo no he escuchado ningún reclamo sobre los pacientes, ellos siempre súper alegres, ¿cómo estás hoy?” (Reino Unido).

\section{CONCLUSIONES Y DISCUSIÓN}

A la luz de los resultados y volviendo la mirada al objetivo planteado al inicio es posible afirmar que la característica cultural que destaca en torno a las prácticas profesionales de los estudiantes en distintos países de la Unión es la "diversidad" y esta característica se interpreta partir de las distintas situaciones experimentadas y relatadas por los estudiantes.

La importancia de este conocimiento radica en que esta situación afecta las oportunidades de aprendizaje de los alumnos. Es decir, los hechos develados revelan que la coexistencia de dos realidades paralelas: un profesional de enfermería muy cualificado junto a un personal técnico con distintos niveles de formación y esta situación afecta la experiencia práctica de los estudiantes al momento de acceder a realizar determinadas actividades cuidadoras. Así, en unos escenarios las prácticas del alumno se centran más en los cuidados básicos y en otros en los cuidados más avanzados. Esto se explica porque la profesionalidad de una profesión pasa por el desarrollo de la cultura profesional y el avance en los niveles de formación, autonomía y compromiso con el trabajo (Fernández Pérez, 1988; García García, 1986; 1996; 2006; Ortega y Valera, 1991) (García García, Emilio; Martínez Pérez, Lourdes; Carvalho da Veiga, Elizabeth; 2007)

Además, se puede deducir de los datos que la cualificación del profesional a cargo del estudiante también interviene en el modo como se desarrolla la experiencia del alumno de enfermería, tal como se describe en relación a la figura del mentor. Esta situación es coherente con las características del buen profesor señaladas por informes de la Organización para la Cooperación y el Desarrollo Económico [OCDE] y estos hechos, además, influyen en la valoración que el estudiante hace de la experiencia de intercambio Erasmus. En este último aspecto también interviene el tipo de cuidado otorgado en su lugar de prácticas, desvelándose como satisfactoria para los estudiantes la experiencia dentro de un entorno de cuidados personalizados. 


\section{REFERENCIAS}

- Arnal, J., del Rincón, D., y Latorre, A. (1992). Nociones básicas sobre investigación. En Investigación Educativa. Fundamentos y Metodología (1 ${ }^{\text {a }}$ ed., págs. 2-23). Barcelona, España: Labor, S.A.

- Barfield, T. (Ed.). (1997, 2000). Diccionario de Antropología. The dictionary of anthropology. (C. S. Rodrigo, Trad.) Bellaterra. Barcelona.

- Boyle, J. S. (1994, 2003). Estilos de etnografía. En U. d. Facultad de Enfermería, \& J. M. Morse (Ed.), Asuntos criticos en los métodos de investigación cualitativa (E. Zimmerman, Trad., $1^{a}$ ed., págs. 197-214). Antioquía, Colombia: Universidad de Antioquía.

- Cabrero J, Richart M. (1996) El debate de la investigación cualitativa frente a la investigación cuantitativa. Enfermería clínica; 6 (5): 212-217.

- Cresswell, M. y Godelier, M. (1981) Útiles de encuesta y de análisis antropológicos. Ed. Fundamentos. Madrid.

- Diario Oficial de la Unión Europea 31.3.2011. Convocatoria de propuestas específica - EAC/16/11.Carta Universitaria Erasmus 2012 (2011/C 99/05) obtenido 712 2011, desde http://eur-lex.europa.eu/LexUriServ/LexUriServ.do?uri=OJ:C:2011:099:0030:0030:ES:PDF

- Decisión no 1720/2006/CE del Parlamento Europeo y del Consejo, de 15 de noviembre de 2006, por la que se establece un programa de acción en el ámbito del aprendizaje permanente. http://eur-lex.europa.eu/lex/LexUriServ/ LexUriServ.do?uri=OJ:L:2006:327:0045:0068:ES:PDF

- Ferraris, M. (2000). Historia de la Hermenéutica.(Pérez de Tudela, J. traductor. Storia dell'Ermeneutica. R: C: S: Libri S.p.A., Milán; Bompiani, 1988). Edicones Akal, S.A. Colmenar Viejo-Madrid.

- Fernández Pérez, M. (1988). La profesionalización del docente. Madrid: Escuela Española.

- Gadamer, H.G. (1960, 1975,1977). Verdad y Método. Fundamentos de una hermenéutica filosófica. (traductor Olasagasti, Manuel. Sígueme, Salamanca. 1992: 63-71). Wahrheit und Methode. 4a edición. Tubinga, Mohr.1975. - García García, E. (1986). Función, Status y Rol del Profesor. En J. Mayor (dir.). Sociología y Psicología Social de la Educación. Madrid: Anaya.
- García García, E. (2006). Las competencias del profesor en la sociedad del conocimiento. En R. Mejía (Coord.). Educación, globalización y desarrollo humano. Santo Domingo, RD: Buho.

- García Marco, Francisco Javier (1995, jul.-dic) Hacia un modelo de intervención en los procesos de transmisión del conocimiento. Scire. 1 (2) págs. 109-118.

- Guba, E., y Lincoln, Y. (1985, 90,94). Paradigmas en competencia en la investigación cualitativa. Por los rincones: antología de métodos en la investigación social, (C. Denman, y J. Haro,

- Hesse, M. (1991) Ciencias de la naturaleza y ciencias del espíritu. En Filosofía de las ciencias humanas y sociales (J. Mardones, $1^{\text {a }}$ ed., págs. 103-106). Barcelona: Anthropos.

- Kerényi, K. (1963,1964). Origene e senso dell'ermeneutica: (O.M. Nobile, em archivio di filosofía, 1-2:129-137). Hermeneia und Hermeneutik. Griechische grundbegriffe, Zurich, págs. 42-52.

- Kottak, C. P. (2006). ¿Qué es la Antropología? En Antropología Cultural. Cultural Anthropology (11 a en español, traducida de la $11^{\text {a }}$ edición en inglés (ed.), págs. 3-21). Madrid: Mc Graw Hill.

- Letelier, M. (2010). Aproximación a la Transmisión - Adquisición de cultura profesional en enfermería: Experiencia de un grupo de enfermeras principiantes graduadas de la Universidad de los Andes, Santiago de Chile. Tesis Suficiencia Investigadora no publicada. Universidad de Alicante.

- Libro Blanco. (2005). Titulo de Grado en Magisterio. Madrid: ANECA.

- Martínez Martín María Luisa, (2007). 30 años de evolución de la formación enfermera en España. Educación Médica; 10 (2): 93-96.

- Ministerio de Educación, Cultura y Deporte, (2003). La integración del sistema universitario español en el Espacio Europeo de Educación Superior. Documento Marco. Madrid.

- Ortega, F.; Varela, A. (1991). La profesión de maestro. Madrid: CIDE.

- Prentice - Hall,inc. (1993). Webster'new world encyclopedia (College ed.revisada, ilustrada ed.). New York.: autor. 
- Real Decreto 1231/2001, de 8 de noviembre, por el que se aprueban los Estatutos Generales de la Organización Colegial de Enfermería de España, del Consejo General y de Ordenación de la actividad profesional de enfermería.

- Real Decreto 1125/2003, de 5 de septiembre, por el que se establece el sistema europeo de créditos y el sistema de calificaciones en las titulaciones universitarias de carácter oficial y validez en todo el territorio español.

- Real Decreto 1044/2003, de 1 de agosto, por el que se establece el procedimiento para la expedición por las universidades del Suplemento Europeo al título.
- Real Decreto 55/2005, de 21 de enero, por el que se establece la estructura de las enseñanzas universitarias y se regulan los estudios universitarios de Grado.

- Real Decreto 56/2005, de 21 de enero, por el que se regulan los estudios universitarios oficiales de Posgrado.

- Valles, M. S. (1999, 2000, 2003, 2007). Variedad de paradigmas y perspectivas en la investigación cualitativa. En Técnicas cualitativas de investigación social. Reflexión metodológica y práctica profesional (cuarta ed., págs. 47-68). Madrid: Síntesis, S.A.

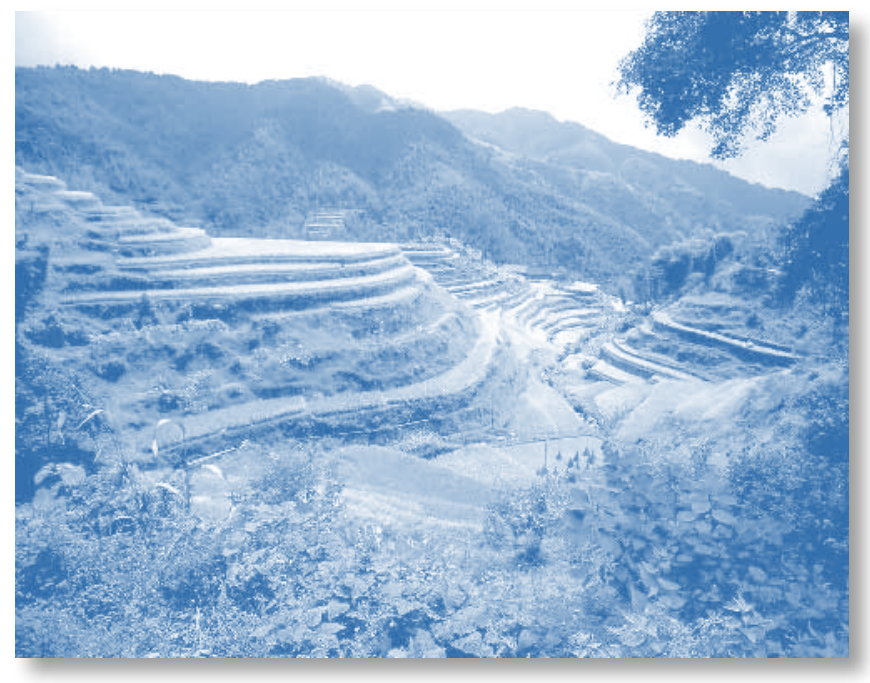

